\title{
Development of UPLC-MS/MS Method for Studying the Pharmacokinetic Interaction Between Dasatinib and Posaconazole in Rats
}

\author{
Suili Yang ${ }^{1, *}$ \\ Xiaoshan Zhang ${ }^{2,3, *}$ \\ Yuzhen Wang,3 \\ Congcong Wen ${ }^{4}$ \\ Chenxiang Wang ${ }^{3}$ \\ Ziye Zhou (D) ${ }^{5}$ \\ Guanyang Lin ${ }^{3}$
}

'Department of Neurology, The First Affiliated Hospital of Wenzhou Medical University, Wenzhou, Zhejiang, People's Republic of China; ${ }^{2}$ College of Pharmacy, Wenzhou Medical University, Wenzhou, Zhejiang, People's Republic of China; ${ }^{3}$ Department of Pharmacy, The First Affiliated Hospital of Wenzhou Medical University, Wenzhou, Zhejiang, People's Republic of China; ${ }^{4}$ Laboratory Animal Center, Wenzhou Medical University, Wenzhou, Zhejiang, People's Republic of China; ${ }^{5}$ Clinical Research Center, The First Affiliated Hospital of Wenzhou Medical University, Wenzhou, Zhejiang, People's Republic of China

*These authors contributed equally to this work

Correspondence: Guanyang Lin; Ziye

Zhou

Department of Pharmacy, The First Affiliated Hospital of Wenzhou Medical University, Nanbaixiang Street, Ouhai

District, Wenzhou City, 325000, People's

Republic of China

Tel +86-577-55579706

Email 13867702133@I63.com;

redd88@163.com
Background and Aim: Dasatinib is approved for the treatment of leukaemia worldwide. Triazole agents such as posaconazole may be used for the control of secondary fungal infection with leukaemia. This work aimed to develop a bioanalytical method to study the potential interaction between dasatinib and posaconazole.

Methods: An ultrahigh-performance liquid chromatography-tandem mass spectrometry method was established to measure the plasma concentrations of dasatinib and posaconazole in rats simultaneously. Simple protein precipitation with acetonitrile was applied to extract dasatinib and posaconazole in samples. The chromatographic separation of analytes was conducted on an UPLC BEH C18 column using a mobile phase consisting of $0.1 \%$ aqueous formic acid and acetonitrile. Dasatinib and posaconazole were monitored in positive ion mode with the following mass transition pairs: $\mathrm{m} / \mathrm{z} 488.2 \rightarrow 401.1$ for dasatinib and $\mathrm{m} / \mathrm{z}$ $701.3 \rightarrow 683.4$ for posaconazole. The method was successfully applied for pharmacokinetic interaction between dasatinib and posaconazole.

Results: The established method expressed good linearity in 1-1000 $\mathrm{ng} / \mathrm{mL}$ of dasatinib and $5-5000 \mathrm{ng} / \mathrm{mL}$ of posaconazole, with limit of detection was $1 \mathrm{ng} / \mathrm{mL}$ and $5 \mathrm{ng} / \mathrm{mL}$, respectively. Methodology validations, including accuracy, precision, matrix effect, recovery, and stability, met the US Food and Drug Administration (FDA) acceptance criteria for bioanalytical method validation. Dasatinib strongly inhibited the clearance of posaconazole in vivo, while posaconazole expressed no significant effect on the pharmacokinetics of dasatinib.

Conclusion: Dasatinib alters the pharmacokinetics of posaconazole. Attention should be paid to the unexpected risk of adverse clinical outcomes when posaconazole is coadministered with dasatinib.

Keywords: dasatinib, posaconazole, UPLC-MS/MS, interaction, pharmacokinetics

\section{Introduction}

Dasatinib is an oral tyrosine kinase inhibitor that targets the chimeric cytoplasmic protein BCR-ABL and downregulates its enzymatic activity to inhibit leukaemogenesis (Figure 1A). It is approved as first-line therapy for chronic myeloid leukaemia (CML) or as an alternative to imatinib therapy in case of intolerance or resistance. ${ }^{1,2}$ It is also commonly used as relapse treatment in the acute phase of CML and Philadelphia chromosome-positive acute lymphoblastic leukaemia, both in adult patients and children., ${ }^{3,4}$ Dasatinib exhibited clinical efficacy in adult patients, associated with 5 -year overall survival of $18 \%-64 \%{ }^{5}$ In children, the 5-year overall survival was $86 \%$ under the treatment of dasatinib. ${ }^{6}$ 

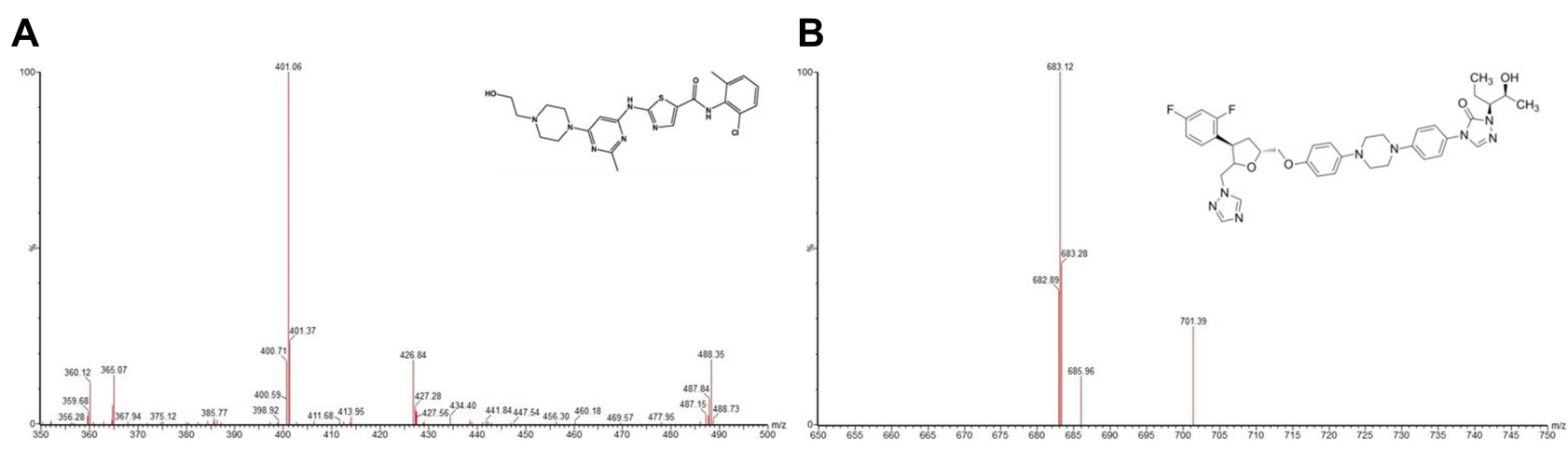

Figure I Mass spectra and structures of dasatinib (A) and posaconazole (B) in present study.

In the clinic, patients with acute leukaemia are considered immunocompromised and face a high risk of developing fungal infections. ${ }^{7,8}$ Triazoles are the standard of care in patients with acute leukaemia receiving remission induction therapy or allogeneic stem cell transplantation. ${ }^{9,10}$ Posaconazole is a second-generation triazole agent with broad-spectrum antifungal activity (Figure 1B). It is labelled for prophylaxis of invasive yeast and mold infections in high-risk immunocompromised patients with leukaemia. ${ }^{11}$

Dasatinib is metabolized in the liver by the CYP450 isoenzyme CYP3A4 and is subject to drug interactions with CYP3A4 inhibitors. ${ }^{12,13}$ Ketoconazole, a classic CYP3A4 inhibitor, increases dasatinib exposure by nearly five-fold in patients, correlated with an increase in corrected QT values of approximately $6 \mathrm{msec}^{14}$ It is suggested that co-administration of dasatinib with other triazoles, most of which were moderate or potent CYP3A4 inhibitors, should be avoided. By contrast, posaconazole is metabolized via UDP glucuronidation in vivo, and it is a substrate for P-glycoprotein as well. ${ }^{15}$ The concentration of posaconazole at a steady-state was reduced by approximately $60 \%$ in a patient with a hematologic malignancy in whom posaconazole was coadministered with the UDP glucuronosyltransferase inducer carbamazepine. ${ }^{16}$ As a substrate of UDP glucuronosyltransferase and membrane transporters, ${ }^{4}$ dasatinib may affect posaconazole exposure. Therefore, the risk of co-administration of these two drugs should be evaluated.

There have been no reported methods for simultaneous determination of dasatinib and posaconazole to the best of our knowledge. Therefore, the objective of this study was to develop and validate a bioanalytical method based on ultrahigh-performance liquid chromatography-tandem mass spectrometry (UPLC-MS/MS) to measure plasma levels of these medications simultaneously and to assess potential drug-drug interaction between them in rats by comparing their pharmacokinetic profiles.

\section{Methods and Materials \\ Materials and Animals}

Dasatinib and posaconazole (both purities $>98 \%$ ) were purchased from Canspec Scientific Instruments Co., Ltd. (Shanghai, China). Diazepam (purity $>98 \%$ ) was purchased from the National Institutes for Food and Drug Control (Beijing, China) and used as an internal standard (IS). LC-MS-grade formic acid and acetonitrile were obtained from Merck KGaA (Darmstadt, Germany). Ultrapure water was prepared using a Milli-Q water purification system (Millipore Corp., Bedford, USA).

Adult male Sprague Dawley rats $(267 \pm 18 \mathrm{~g})$ were purchased from the Laboratory Animal Centre of Wenzhou Medical University and housed at $25{ }^{\circ} \mathrm{C}$ and $40-60 \%$ relative humidity under a 12 -h light-dark cycle. The rats were acclimatized for 7 days under laboratory conditions to minimize all possible sources of animal suffering prior to initiation of the experiment. All experimental animal protocols were approved by the Animal Care and Use Committee of Wenzhou Medical University and were carried out following the guidelines for the ethical review of laboratory animal welfare People's Republic of China National Standard GB/T 35892-2018.

\section{Instrumentations and Analytical \\ Conditions}

The UPLC-MS/MS system consisted of the Waters ACQUITY UPLC I-Class system and Waters Xevo TQ-S triple quadrupole tandem mass spectrometer equipped with an electrospray ionization source (Milford, USA). All experimental data were acquired in centroid mode and 
processed using MassLynx 4.1 software and the TargetLynx programme (Milford, USA).

Chromatographic analysis was performed using a $\mathrm{BEH}$ C18 column $(2.1 \times 100 \mathrm{~mm}, 1.7 \mu \mathrm{m}$; Waters, USA $)$ with gradient elution. The mobile phase was made up of $0.1 \%$ aqueous formic acid and acetonitrile. The gradient programme was set as Table 1. The volume of sample injection was set at $1.0 \mu \mathrm{L}$. The autosampler was maintained at $10^{\circ} \mathrm{C}$, while the temperature of the column was set at $40{ }^{\circ} \mathrm{C}$.

Analytes and IS were determined in multiple reaction monitoring modes with positive ion pairs: $\mathrm{m} / \mathrm{z}$ $488.2 \rightarrow 401.1$ for dasatinib and $\mathrm{m} / \mathrm{z} \quad 701.3 \rightarrow 683.4$ for posaconazole; $\mathrm{m} / \mathrm{z} 285.2 \rightarrow 154$.1 for IS. The cone voltage and collision energies were $30 \mathrm{~V}$ and $26 \mathrm{eV}$ for dasatinib, $80 \mathrm{~V}$ and $30 \mathrm{eV}$ for posaconazole, and $10 \mathrm{~V}$ and $30 \mathrm{eV}$ for IS.

\section{Preparation of the Standard Solutions}

Stock solutions of dasatinib, posaconazole, and IS were dissolved to $1.0 \mathrm{mg} / \mathrm{mL}$ in methanol. The working solution was prepared by corresponding serial dilutions from the stock solution. Calibration standard and quality control (QC) samples were prepared by spiking blank plasma with appropriate volumes of the working solution. For the determination of analytes in plasma, the concentrations of calibration standards for dasatinib were set at 1, 10, 20, 100,200 , and $1000 \mathrm{ng} / \mathrm{mL}$, and the concentrations of calibration standards for posaconazole were set at 5, 50, $100,500,1000$, and $5000 \mathrm{ng} / \mathrm{mL}$. The concentrations of plasma QC samples were produced at $1 \mathrm{ng} / \mathrm{mL}$ (lower limit of quantification, LLOQ), $3 \mathrm{ng} / \mathrm{mL}$ (LOQ), $500 \mathrm{ng} /$ $\mathrm{mL}$ (MOQ), and $750 \mathrm{ng} / \mathrm{mL}$ (HOQ) for dasatinib. For posaconazole, LLOQ, LOQ, MOQ, and HOQ were set at $5,15,2500,3750 \mathrm{ng} / \mathrm{mL}$, respectively.

Table I Gradient Elution Programme of HPLC for Dasatinib and Posaconazole

\begin{tabular}{|l|l|l|l|}
\hline Time & $\begin{array}{l}\text { O.1\% Aqueous } \\
\text { Formic Acid } \\
\text { (Composition, \%) }\end{array}$ & $\begin{array}{l}\text { Acetonitrile } \\
\text { (Composition, \%) }\end{array}$ & $\begin{array}{l}\text { Flow } \\
\text { Rate }\end{array}$ \\
\hline 0 & 90 & 10 & $0.3 \mathrm{~mL} / \mathrm{min}$ \\
0.5 & 90 & 10 & \\
1.0 & 10 & 90 & \\
2.0 & 10 & 90 & \\
2.5 & 90 & 10 & \\
3.0 & 90 & 10 & \\
\hline
\end{tabular}

\section{Sample Preparation}

A $100 \mu \mathrm{L}$ aliquot of plasma sample was placed in an Eppendorf tube, and $300 \mu \mathrm{L}$ acetonitrile containing 100 $\mathrm{ng} / \mathrm{mL}$ IS was added, vortex mixed for $1 \mathrm{~min}$, and centrifuged at $13,000 \mathrm{rpm}$ and $4{ }^{\circ} \mathrm{C}$ for $10 \mathrm{~min}$. Then the $100 \mu \mathrm{L}$ supernatants were transferred to sample bottles, and $1-\mu \mathrm{L}$ aliquots of the supernatant were injected for UPLC-MS /MS analysis.

\section{Method Validation}

According to the US Food and Drug Administration (FDA) guidance for bioanalytical method validation, the method validations including calibration curve, selectivity, accuracy, precision, matrix effect, recovery, and stability were performed to evaluate the method.

\section{Pharmacokinetic Interaction Study}

Twenty-four rats were randomly divided into three groups: group A (dasatinib, $10 \mathrm{mg} / \mathrm{kg}$ ); group B (posaconazole, $40 \mathrm{mg} / \mathrm{kg}$ ); and group C (dasatinib $10 \mathrm{mg} / \mathrm{kg}$ plus posaconazole $40 \mathrm{mg} / \mathrm{kg}$ ), eight rats per group. The rats fasted for $12 \mathrm{~h}$ before the experiment, and only water was allowed. When the experiment was initiated, dasatinib and posaconazole dissolved in $0.1 \%$ sodium carboxymethyl cellulose (CMC-Na) orally administered to the groups $\mathrm{A}$ and $\mathrm{B}$ rats, respectively. Group $\mathrm{C}$ rats received dasatinib and posaconazole mixed $\mathrm{CMC}-\mathrm{Na}$ solution. Blood samples were directly collected into heparin tubes via the tail vein at $0.083,0.25,0.5,0.75,1,1.25,1.5,2,3,4,5,6,8,10,12$, 24,48 , and $72 \mathrm{~h}$ after administration. The plasma samples were obtained from the blood by centrifugation at $3500 \mathrm{rpm}, 4{ }^{\circ} \mathrm{C}$ for $10 \mathrm{~min}$, and $100 \mu \mathrm{L}$ was transferred to fresh tubes. All samples were stored at $-80{ }^{\circ} \mathrm{C}$ until use.

\section{Statistical Analysis}

The pharmacokinetic parameters were calculated using DAS software 3.0 (Bontz Inc., Beijing, China) with noncompartmental analysis. The mean concentration-time curves were constructed using GraphPad Prism 8.0 (GraphPad Software Inc., San Diego, USA). The nonparametric Mann-Whitney $U$-test was used to evaluate pharmacokinetics' difference between dasatinib or posaconazole treatment alone group with two drugs combined treatment group by SPSS software 23.0 (IBM Corp., Chicago, USA). Pharmacokinetic profiles were expressed as mean $\pm \mathrm{SD}$ and pharmacokinetic parameters were presented as median (min-max). $\mathrm{P}<0.05$ represented statistical significance. 


\section{Results}

\section{Method Validation}

\section{Selectivity}

The specificity of the method was evaluated by comparing the chromatograms of blank plasma from six rats with those spiked with the LLOQ of dasatinib, posaconazole, and IS. There was no interference from the endogenous substances at the dasatinib, posaconazole, and IS retention times, which were 1.67, 1.95, and 2.06 min (Figure 2), respectively.

\section{Calibration Curve and LLOQ}

A weighted $\left(1 / \mathrm{x}^{2}\right)$ linear regression of the peak area ratio of analyte versus IS to the analyte concentration was generated to define the calibration curve. The linear relationships $\mathrm{r}^{2}$ for the determination of two drugs in this bioanalytical assay were no less than 0.99 , and the regression equations were $\mathrm{R}=0.0734 \mathrm{C}-0.0281$ for dasatinib and $\mathrm{R}=0.0233 \mathrm{C}+0.0256$ for posaconazole, where $\mathrm{C}$ indicates the nominal concentration level of the analyte, and $\mathrm{R}$ indicates the peak area ratio of analyte to the IS. The analytical signal of the analyte at the LLOQ was more than ten times of noise from the blank sample at the same retention time.

\section{Precision and Accuracy}

Six replicates of LLOQ, LOQ, MOQ, and HOQ samples were evaluated over three consecutive days to calculate the inter-day accuracy and precision. They were evaluated on the same day to calculate the intra-day accuracy and precision. A summary of the data is provided in Table 2. The intra- and inter-day accuracy of dasatinib in plasma were between $-2.53 \%$ and $9.08 \%$, and the intra- and inter-day precisions were all less than $10 \%$. The intra- and inter-day accuracy of posaconazole in plasma were between $2.51 \%$ and $8.12 \%$, and the intra- and inter-day precisions were all less than $11 \%$. The precision and accuracy results met the requirements of FDA principles, ie, they should be within $\pm 15 \%$ ( $\pm 20 \%$ for LLOQ) and below $15 \%$ ( $20 \%$ for LLOQ), respectively.

\section{Matrix Effect and Extraction Recovery}

The matrix effect was evaluated by comparing the analyte's peak areas in the processed blank plasma samples with those of the analyte in methanol at the corresponding concentration levels. The extraction recovery was assessed by comparing the analyte chromatographic peak areas in the QC plasma samples with those of the analyte added in the processed blank plasma samples at the corresponding concentration levels. Six replicates of LOQ, MOQ, and HOQ levels were performed to assess the matrix effect and extraction recovery of dasatinib and posaconazole in plasma. A summary of the data is provided in Table 2 . The matrix effect of dasatinib was calculated to range from $88.71 \%$ to $94.19 \%$. The posaconazole was between $91.92 \%$ and $100.96 \%$, indicating no significant ion enhancement or suppression effect from the endogenous materials in plasma during the entire assessment procedure. The average extraction recoveries of dasatinib and posaconazole in plasma were $88.88 \%$ and $80.59 \%$, respectively.

\section{Stability}

Stabilities of dasatinib and posaconazole were assessed under various storage and processing conditions at LOQ and HOQ concentrations. As shown in Table 3, the dasatinib and posaconazole were stable in plasma at ambient temperature for $12 \mathrm{~h}, 6 \mathrm{~h}$ in autosampler after preparation, three freeze-thaw cycles, and 30 days at $-80{ }^{\circ} \mathrm{C}$.

\section{Pharmacokinetic Interaction Study}

The newly established UPLC-MS/MS method for concentration determination of dasatinib and posaconazole in plasma was performed to evaluate the pharmacokinetic interaction between two medications in rats. After oral administration of dasatinib alone, posaconazole alone or their combination, the mean concentrations versus time profiles in the three groups were illustrated in Figure 3. The primary pharmacokinetic parameters are listed in Table 4. When dasatinib was co-administered with posaconazole, neither the pharmacokinetic parameters nor pharmacokinetic profiles of dasatinib showed significant differences with the dasatinib alone group. However, the parameters of posaconazole in the combined treatment group, including $\mathrm{AUC}_{0-\mathrm{t}}, \mathrm{AUC}_{0-\infty}$, and $\mathrm{C}_{\max }$, increased significantly by 8.81 -fold, 9.35 -fold, and 4.97 -fold, respectively $(p<0.01)$. The median $T_{\max }$ of posaconazole increased from $8 \mathrm{~h}$ to $24 \mathrm{~h}$, and $\mathrm{CL}_{\mathrm{z} / \mathrm{F}}$ decreased substantially to $9.34 \%$ of the treatment alone group $(\mathrm{P}<0.01)$.

\section{Discussion}

A method for the simultaneous determination of dasatinib and posaconazole was established. For specificity and sensitivity maximization of the two analytes, the mass spectrometric conditions were optimized. More than three product ion transitions of each analyte were 


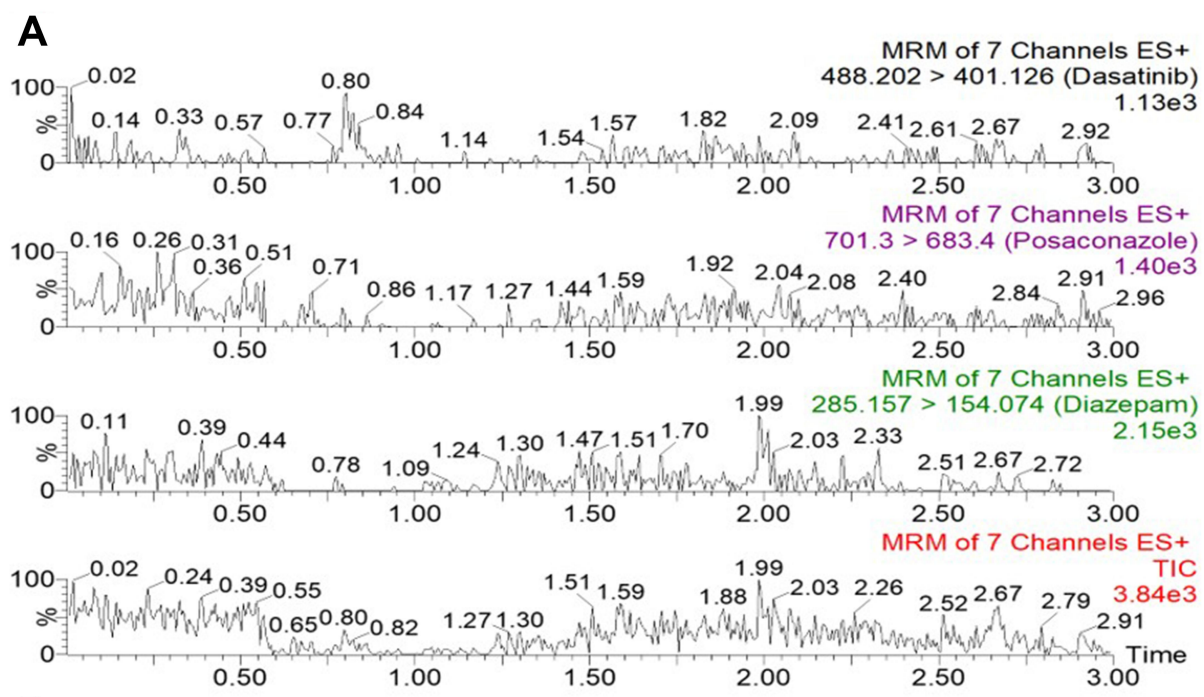

B
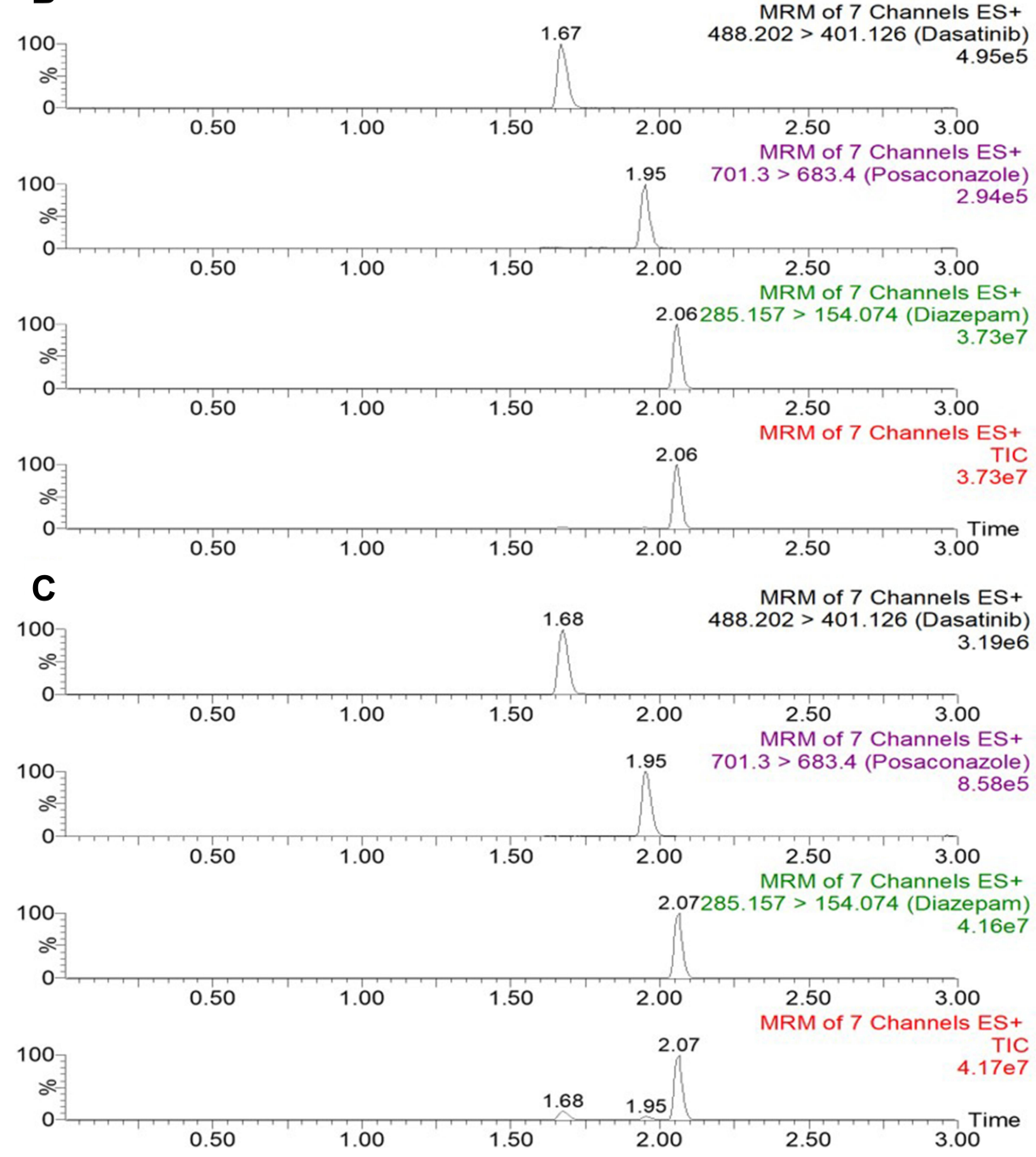

Figure 2 Representative liquid chromatograms of dasatinib, posaconazole, and IS in rat plasma sample. (A) blank plasma; (B) plasma standard of I0 ng/mL dasatinib and 50 $\mathrm{ng} / \mathrm{mL}$ posaconazole; (C) plasma sample from combined treatment group $5 \mathrm{~h}$ after orally administration of dasatinib and posaconazole simultaneously. 
Table 2 Accuracy, Precision, Matrix Effect and Recovery for the Analysis of Dasatinib and Posaconazole in Rat Plasma ( $n=6$ )

\begin{tabular}{|c|c|c|c|c|c|c|c|}
\hline \multirow[t]{2}{*}{ Analyte } & \multirow[t]{2}{*}{ Concentration $(\mathrm{ng} / \mathrm{mL})$} & \multicolumn{2}{|c|}{ Accuracy (RE, \%) } & \multicolumn{2}{|c|}{ Precision (RSD, \%) } & \multirow[t]{2}{*}{ Matrix Effect (\%) } & \multirow[t]{2}{*}{ Recovery (\%) } \\
\hline & & Intra-Day & Inter-Day & Intra-Day & Inter-Day & & \\
\hline \multirow[t]{4}{*}{ Dasatinib } & I & 3.17 & 2.97 & 7.99 & 9.17 & - & - \\
\hline & 3 & 5.84 & -2.53 & 7.65 & 7.45 & $88.7 I \pm 2.54$ & $89.87 \pm 4.32$ \\
\hline & 500 & 9.08 & 6.86 & 2.69 & 3.54 & $94.19 \pm 6.9$ & $87.85 \pm 2.32$ \\
\hline & 750 & 5.23 & 7.06 & 5.36 & 3.85 & $92.47 \pm 7.24$ & $88.93 \pm 3.27$ \\
\hline \multirow[t]{4}{*}{ Posaconazole } & 5 & 7.29 & 4.21 & 6.74 & 10.11 & - & - \\
\hline & 15 & 6.36 & 7.39 & 7.44 & 6.93 & $91.92 \pm 8.41$ & $86.20 \pm 8.02$ \\
\hline & 2500 & 6.31 & 8.12 & 6.48 & 3.53 & $100.96 \pm 5.86$ & $78.50 \pm 9.09$ \\
\hline & 3750 & 2.51 & 4.34 & 8.53 & 3.88 & $98.57 \pm 7.74$ & $77.06 \pm 8.40$ \\
\hline
\end{tabular}

Table 3 The stability for the Analysis of Dasatinib and Posaconazole in Rat Plasma $(n=6)$

\begin{tabular}{|l|l|l|l|l|l|}
\hline \multirow{2}{*}{ Analytes } & Concentration (ng/mL) & \multicolumn{5}{|l|}{ Stability (\%) } \\
\cline { 3 - 6 } & & Ambient, I 2 h & Autosampler, 6 h & Three Freeze-Thaw Cycles & $-80{ }^{\circ}$ C, 30 Days \\
\hline Dasatinib & 3 & 7.42 & 8.42 & 5.35 & 5.12 \\
& 750 & 6.03 & 3.92 & 4.68 & 5.88 \\
\hline Posaconazole & 15 & 3.96 & 5.6 & 7.76 & 6.21 \\
& 3750 & 5.82 & 4.2 & 4.14 & 2.95 \\
\hline
\end{tabular}

compared to produce the highest sensitivity of analytes. Furthermore, optimized cone voltage and collision energy were set for the intensity and relative abundance of precursor ions and fragment ions. Dasatinib and posaconazole were found to yield a higher response in positive mass mode than in negative mode. The most abundant fragment ions for multiple reaction monitoring were $\mathrm{m} / \mathrm{z} \quad 488.2 \rightarrow 401.1$ and $\mathrm{m} / \mathrm{z} \quad 701.3 \rightarrow 683.4$, with cone voltage values of $30 \mathrm{~V}$ and $80 \mathrm{~V}$, collision energy values of $26 \mathrm{eV}$ and $30 \mathrm{eV}$ for dasatinib and posaconazole, respectively.

Several studies described the LC-MS/MS-based detection method of dasatinib in plasma. ${ }^{17-19}$ Although these studies developed determination methods for dasatinib with high sensitivity and accuracy, liquid-liquid extraction and solid-phase extraction were chosen for sample
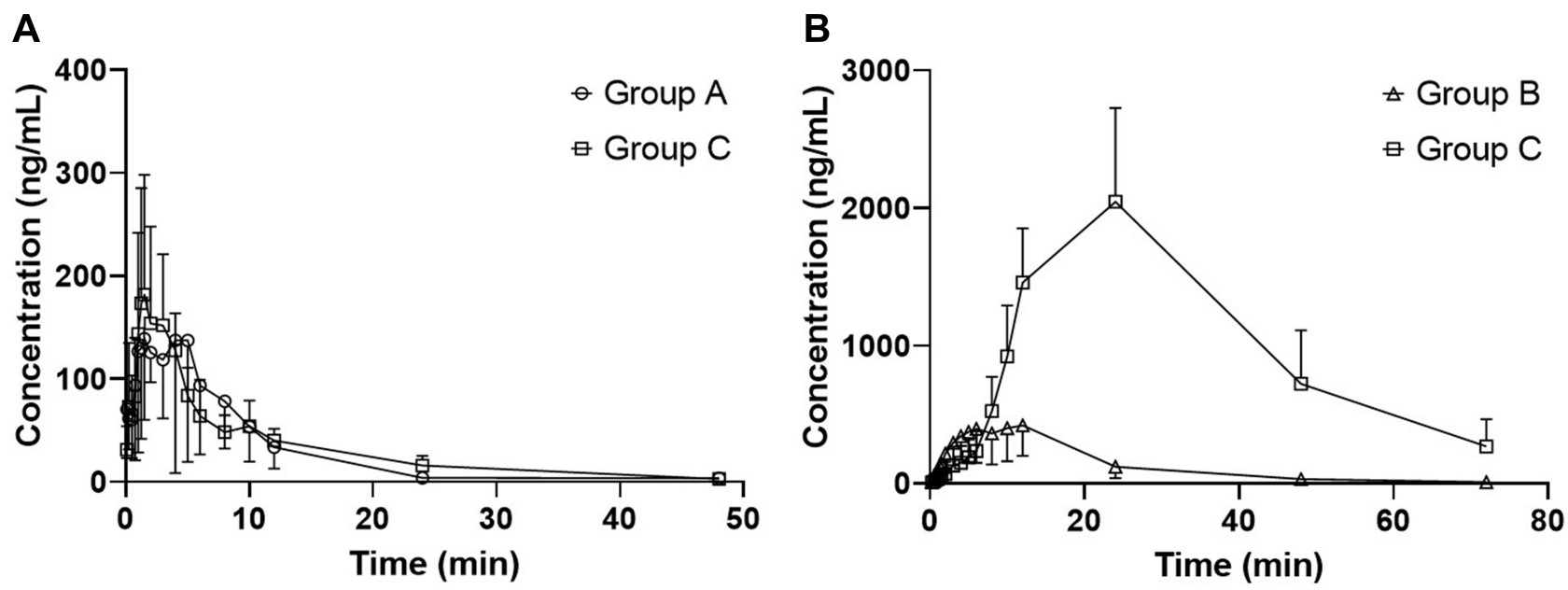

Figure 3 Mean concentration-time curves of dasatinib and posaconazole in dasatinib treatment alone group, posaconazole treatment alone group, and two drugs combined treatment group (A and B). Group A: $10 \mathrm{mg} / \mathrm{kg}$ dasatinib; group B: $40 \mathrm{mg} / \mathrm{kg}$ posaconazole; group C: $10 \mathrm{mg} / \mathrm{kg}$ dasatinib plus $40 \mathrm{mg} / \mathrm{kg}$ posaconazole. Each time-point represents the mean \pm SD. 
Table 4 The Main Pharmacokinetic Parameters of Dasatinib and Posaconazole in Three Groups ( $\mathrm{n}=8$ Each Group)

\begin{tabular}{|c|c|c|c|c|}
\hline \multirow[t]{2}{*}{ Parameters } & \multirow[t]{2}{*}{ Group A } & \multirow[t]{2}{*}{ Group B } & \multicolumn{2}{|l|}{ Group C } \\
\hline & & & Dasatinib & Posaconazole \\
\hline $\mathrm{AUC}_{0-\mathrm{t}}(\mathrm{ng} * \mathrm{~h} / \mathrm{mL})$ & I350.86 (878.02-2376.38) & $7528.97(4217.76-17,907.69)$ & | 395.6 (|059.6-|905.2) & $73,856.23(25,260.08-97,420.83)^{* *}$ \\
\hline$A \cup C_{0-\infty}\left(n g^{*} h / m L\right)$ & I37|.08 (878.2-2376.44) & 7617.16 (4533.24-18,087.2) & $1677.42(|| 55.2-2 \mid 85.62)$ & $78,809.65(26,028.33-121,006.9)^{* *}$ \\
\hline$t_{1 / 2 z}(h)$ & $4.52(3.04-14)$ & $12.43(9.63-23.75)$ & $9.63(3.06-19.92)$ & $15.25(10.72-24.33)$ \\
\hline $\mathrm{T}_{\max }(\mathrm{h})$ & $1.75(I-5)$ & $8(4-12)$ & $1.375(I-3)$ & $24(12-24)^{* *}$ \\
\hline $\mathrm{CL}_{\mathrm{z} / \mathrm{F}}(\mathrm{L} / \mathrm{h} / \mathrm{kg})$ & $7.36(4.21-11.39)$ & $5.46(2.21-8.82)$ & $5.97(4.58-8.66)$ & $0.5 \mathrm{I}(0.33-1.54)^{* *}$ \\
\hline $\mathrm{C}_{\max }(\mathrm{ng} / \mathrm{mL})$ & $189.72(108.48-399.19)$ & $390.27(204.63-892.45)$ & |75.9| (|31.62-403.32) & $2329.47(1119.42-2680.17)^{* *}$ \\
\hline
\end{tabular}

Note: $*^{*} p<0.01$, significant in comparison with group $A$ or group $B$.

Abbreviations: $A_{U} C_{0-t}$, area under the curve from time zero to the last quantifiable concentration; $A U C_{0-\infty}$, area under the curve from time zero to infinity; $t_{1 / 2 z}$, half-life; $\mathrm{T}_{\max }$, time to maximum concentration; $\mathrm{CL}_{\mathrm{z} / \mathrm{F}}$ clearance; $\mathrm{C}_{\max }$, maximal plasma concentration.

preparation. These were expensive and required long times. Another two studies established the mass spectrometry method of posaconazole in human plasma. ${ }^{16,20}$ Because these two methods were applied for pharmacodynamics and toxicity evaluation, the LLOQ settings were not low enough for pharmacokinetic study. Therefore, in addition to lower LLOQ, we sought to shorten the analytical time as well. To accomplish this, we used a gradient elution programme for chromatographic analysis of three analytes. Dasatinib, posaconazole, and diazepam could be separated absolutely in 3.0 min with a symmetrical peak. A rapid and straightforward plasma protein precipitation with acetonitrile was applied for sample preparation, which is suitable for subsequent experiments such as the pharmacokinetic study and met the requirements for highthroughput detection. Additionally, $0.1 \%$ formic acid was added to water to optimize the mobile phase and enhance the mass spectrometric signal intensities of analytes in this study. The LLOQs of dasatinib and posaconazole in this study was set at $1 \mathrm{ng} / \mathrm{mL}$ and $5 \mathrm{ng} / \mathrm{mL}$, respectively.

The newly established UPLC-MS/MS method was successfully applied for concentration determination of dasatinib and posaconazole in plasma to evaluate the pharmacokinetic interaction between two medications in rats. When dasatinib was co-administered with posaconazole, neither the pharmacokinetic parameters nor pharmacokinetic profiles of dasatinib showed significant differences with the dasatinib alone group. This finding suggests that posaconazole did not affect the pharmacokinetics of dasatinib at a clinically relevant dosage. The dose of dasatinib needs not to be adjusted when administered concomitantly with posaconazole. However, the parameters of posaconazole in the combined treatment group changed significantly. This finding suggests that dasatinib expressed a significant inhibitory effect on the metabolism of posaconazole in vivo. This may due to the substrate competition caused by dasatinib. Dasatinib is a substrate of UDP glucuronosyltransferase, which is the same as posaconazole. Dasatinib may completely bind to UDP glucuronosyltransferase and cause the delayed metabolism of posaconazole in vivo. Therefore, unexpected risk of clinical outcomes may occur when posaconazole is coadministered with dasatinib, and dose adjustment should be considered in case of side effects.

\section{Conclusions}

The method established in this study to determine dasatinib and posaconazole simultaneously in rat plasma was simple, fast, and accurate. This bioanalytical method was successfully applied for the evaluation of pharmacokinetic interaction between dasatinib and posaconazole in rats. Dasatinib significantly increased the exposure to posaconazole in vivo. Therefore, the combination use of posaconazole with dasatinib should be monitored to avoid the occurrence of side effects.

\section{Acknowledgments}

This work was supported by the Project Supported by Zhejiang Provincial Natural Science Foundation of China [grant number LYY19H310007] and Medical Health Science and Technology Project of Zhejiang Provincial Health Commission [grant number 2018KY519].

\section{Disclosure}

The authors declare no conflicts of interest.

\section{References}

1. Talpaz M, Shah NP, Kantarjian H, et al. Dasatinib in imatinib-resistant Philadelphia chromosome-positive leukemias. New Engl J Med. 2006;354(24):2531-2541. doi:10.1056/NEJMoa055229 
2. Shah NP, Tran C, Lee FY, Chen P, Norris D, Sawyers CL. Overriding imatinib resistance with a novel ABL kinase inhibitor. Science. 2004;305(5682):399-401. doi:10.1126/science.1099480

3. Keating GM. Dasatinib: a Review in Chronic Myeloid Leukaemia and $\mathrm{Ph}$ plus Acute Lymphoblastic Leukaemia. Drugs. 2017;77 (1):85-96. doi:10.1007/s40265-016-0677-x

4. Korashy HM, Rahman AFMM, Kassem MG. Dasatinib. Profiles Drug Subst Excip Relat Methodol. 2014;205-237. doi:10.1016/ B978-0-12-800173-8.00004-0

5. Lennmyr E, Karlsson K, Ahlberg L, et al. Survival in adult acute lymphoblastic leukaemia (ALL): a report from the Swedish ALL Registry. Eur J Haematol. 2019;103(2):88-98. doi:10.1111/ejh. 13247

6. Slayton WB, Schultz KR, Kairalla JA, et al. Dasatinib plus intensive chemotherapy in children, adolescents, and young adults with philadelphia chromosome-positive acute lymphoblastic leukemia: results of Children's Oncology Group Trial AALL0622. J Clin Oncol. 2018;36(22):2306-2314. doi:10.1200/JCO.2017.76.7228

7. Mariette C, Tavernier E, Hocquet D, et al. Epidemiology of invasive fungal infections during induction therapy in adults with acute lymphoblastic leukemia: a GRAALL-2005 study. Leukemia Lymphoma. 2017;58(3):586-593. doi:10.1080/10428194.2016.1204652

8. Keng MHB, Keng HLC, Tan BH, Wong GC. High risk of invasive fungal infections in adult acute lymphoblastic leukemia patients receiving induction and salvage chemotherapy. Leukemia Lymphoma. 2017;58(8):2017-2018. doi:10.1080/10428194.2016.126 7351

9. Cornely OA, Robertson MN, Haider S, et al. Pharmacokinetics and safety results from the Phase 3 randomized, open-label, study of intravenous posaconazole in patients at risk of invasive fungal disease. J Antimicrob Chemoth. 2017;72(12):3501-3413. doi:10.1093/jac/dkx382

10. Girmenia C, Busca A, Candoni A, et al. Breakthrough invasive fungal diseases in acute myeloid leukemia patients receiving mould active triazole primary prophylaxis after intensive chemotherapy: an Italian consensus agreement on definitions and management. Med Mycol. 2019;57:S127-S137. doi:10.1093/mmy/myy091

11. Wong TY, Loo YS, Veettil SK, et al. Efficacy and safety of posaconazole for the prevention of invasive fungal infections in immunocompromised patients: a systematic review with meta-analysis and trial sequential analysis. Sci Rep-Uk. 2020;10(1):14575. doi:10.1038/ s41598-020-71571-0
12. Wang LF, Christopher LJ, Cui DH, et al. Identification of the human enzymes involved in the oxidative metabolism of dasatinib: an effective approach for determining metabolite formation kinetics. Drug Metab Dispos. 2008;36(9):1828-1839. doi:10.1124/dmd.107.020255

13. Li XH, He YJ, Ruiz CH, Koenig M, Cameron MD. Characterization of dasatinib and its structural analogs as CYP3A4 mechanism-based inactivators and the proposed bioactivation pathways. Drug Metab Dispos. 2009;37(6):1242-1250. doi:10.1124/dmd.108.025932

14. Johnson FM, Agrawal S, Burris H, et al. Phase 1 pharmacokinetic and drug-interaction study of dasatinib in patients with advanced solid tumors. Cancer-Am Cancer Soc. 2010;116(6):1582-1591. doi: $10.1002 /$ cncr. 24927

15. Lin JH. Drug-drug interaction mediated by inhibition and induction of P-glycoprotein. Adv Drug Deliver Rev. 2003;55(1):53-81. doi:10.1016/S0169-409X(02)00171-0

16. Chae H, Cho SY, Yu H, et al. Determination of posaconazole concentration with LC-MS/MS in adult patients with hematologic malignancy. Clin Chim Acta. 2015;450:220-226. doi:10.1016/j. cca.2015.08.023

17. Alzoman NZ, Maher HM, Shehata SM, Abanmy NO. UPLC-MS/MS study of the effect of dandelion root extract on the plasma levels of the selected irreversible tyrosine kinase inhibitors dasatinib, imatinib and nilotinib in rats: potential risk of pharmacokinetic interactions. Biomed Chromatogr. 2019;33(12):e4674. doi:10.1002/bmc.4674

18. Maher HM, Alzoman NZ, Shehata SM, Abanmy NO. Validated UPLC-MS/MS method for the quantification of dasatinib in plasma: application to pharmacokinetic interaction studies with nutraceuticals in Wistar rats. PLoS One. 2018;13(6):e0199208. doi:10.1371/journal. pone.0199208

19. Zeng J, Cai HL, Jiang ZP, et al. A validated UPLC-MS/MS method for simultaneous determination of imatinib, dasatinib and nilotinib in human plasma. J Pharm Anal. 2017;7(6):374-380. doi:10.1016/j. jpha.2017.07.009

20. Basu SS, Petrides A, Mason DS, Jarolim P. A rapid UPLC-MS/MS assay for the simultaneous measurement of fluconazole, voriconazole, posaconazole, itraconazole, and hydroxyitraconazole concentrations in serum. Clin Chem Lab Med. 2017;55(6):836-844. doi:10. 1515/cclm-2016-0418

\section{Publish your work in this journal}

Drug Design, Development and Therapy is an international, peerreviewed open-access journal that spans the spectrum of drug design and development through to clinical applications. Clinical outcomes, patient safety, and programs for the development and effective, safe, and sustained use of medicines are a feature of the journal, which has also been accepted for indexing on PubMed Central. The manuscript management system is completely online and includes a very quick and fair peer-review system, which is all easy to use. Visit http://www. dovepress.com/testimonials.php to read real quotes from published authors. 